\title{
Glomerular filtration rate in patients with obstructive sleep apnea: the influence of cystatin-C-based estimations and comorbidity
}

\author{
Adam Nowiński ${ }^{1}$, Anna Czyżak-Gradkowska ${ }^{1}$, Luiza Jonczak ${ }^{1}$, Damian Korzybski ${ }^{1}$, Joanna Peradzyńska ${ }^{2}$, \\ Robert Pływaczewski ${ }^{1}$, Paweł Śliwiński ${ }^{1}$ \\ ${ }^{1}$ 2nd Department of Respiratory Medicine, Institute of Tuberculosis and Lung Diseases, Warsaw, Poland; ${ }^{2}$ Department of Epidemiology and \\ Biostatistics, Medical University of Warsaw, Warsaw, Poland \\ Contributions: (I) Conception and design: All authors; (II) Administrative support: A Nowiński; (III) Provision of study materials or patients: All \\ authors; (IV) Collection and assembly of data: All authors; (V) Data analysis and interpretation: All authors; (VI) Manuscript writing: All authors; (VII) \\ Final approval of manuscript: All authors. \\ Correspondence to: Adam Nowiński, MD, PhD. 2nd Department of Respiratory Medicine, Institute of Tuberculosis and Lung Diseases, ul. Plocka 26, \\ 01-138 Warsaw, Poland. Email: a.nowinski@igichp.edu.pl.
}

Background: Recent studies indicate that chronic kidney disease (CKD) is a comorbidity in patients with obstructive sleep apnea (OSA). We hypothesized that the use of the classical muscle-dependent, creatininebased equation to estimate glomerular filtration rate (GFR) in patients with OSA may be inaccurate due to the extreme body mass index (BMI) of some patients. The aim of this study was to establish the role of cystatin-C-based estimation of GFR for the detection of CKD in patients with OSA and typical comorbidities.

Methods: Two hundred and forty consecutive patients with newly diagnosed OSA were enrolled into this cross-sectional study. In all patients estimated GFR (eGFR) was calculated with chronic kidney diseaseepidemiology collaboration group (CKD-EPI) equations using creatinine and cystatin-C. All patients were examined for comorbidities.

Results: In obese patients with OSA significant differences between GFR estimations based on creatinine and cystatin were found: eGFR based on muscle-dependent creatinine measurement was significantly higher than the muscle-independent eGFR based on cystatin-C measurement.

Conclusions: GFR can be routinely screened for using creatinine-based estimations (eGFRcreat). In a selected group of patients with OSA with BMI over $30 \mathrm{~kg} / \mathrm{m}^{2}$ the addition of cystatin-C for assessment of eGFR is suggested.

Keywords: Comorbidity; cystatin-C; glomerular filtration rate (GFR); sleep apnea

Submitted Jul 14, 2019. Accepted for publication Jan 20, 2020.

doi: $10.21037 /$ jtd.2020.02.11

View this article at: http://dx.doi.org/10.21037/jtd.2020.02.11

\section{Introduction}

Obstructive sleep apnea (OSA) is characterized by intermittent hypoxemia during sleep (1). Hypoxia is considered a potential initiator of the processes leading to progressive renal dysfunction (2). Many important comorbidities of OSA, such as hypertension and diabetes, have been identified so far $(3,4)$, but the role of OSA in the development of chronic kidney disease (CKD) is still not clear. Recent studies indicate that CKD is a comorbidity in patients with OSA, although the results of research have not always been in agreement (5). Technical considerations regarding proper measurement or estimation of glomerular filtration rate (GFR) and the influence of comorbidities on the estimated GFR (eGFR) in patients with OSA are possible reasons for these diverse study results (6).

The Kidney Disease Improving Global Outcome Group (KDIGO) suggests the use of serum creatinine for initial 
assessment of eGFR. The KDIGO also suggests performing additional tests, such as measurement of cystatin-C, for confirmatory kidney testing in circumstances in which eGFR based on serum creatinine is less accurate (7). According to the chronic kidney disease-epidemiology collaboration group (CKD-EPI) such specific circumstances are clinical scenarios when patients present with extreme size and extreme muscle mass (8). Both very high and very low muscle mass affect the generation of creatinine, which could affect the calculation of GFR (8). Patients with OSA often present with extreme body mass and size (9). Muscle mass does not necessary correspond directly with body mass index (BMI) in such patients (10), and in the majority of patients with OSA a shift from fat-free muscle mass to higher fat mass index is observed. All such factors added to the multiple comorbidities observed in patients with OSA can lead to clinical dilemmas as to whether to use creatinine- or cystatin-C-based equations when kidney disease is suspected. Almost all studies on assessment of CKD in OSA used classical creatinine-based equations (5,11-15). Only a few studies used cystatin-C for GFR assessment of CKD in patients with OSA (16-18).

We hypothesized that the use of the classical muscledependent, creatinine-based equation to estimate GFR in patients with OSA may be inaccurate due to the extreme BMI of some patients with OSA. Thus, we aimed to establish the role of cystatin-C-based eGFR in patients with OSA and typical comorbidities.

\section{Methods}

This cross-sectional study was performed in the National Institute of Tuberculosis and Lung Disease in Warsaw, at the Sleep Laboratory. During the 2-year study (2011-2012), 240 consecutive patients with newly diagnosed OSA were enrolled.

All patients were assessed according to the current guidelines of the American Academy of Sleep Medicine (AASM) and the Polish Society of Lung Disorders, and the diagnosis of OSA was based on fully attended nocturnal polysomnography (19). All study participants were carefully examined for comorbidities. A comorbidity is defined as a disease coexisting with the primary disease of interest, as suggested by $\operatorname{Sin}$ et al. (20). Diagnoses were made by a group of respiratory and internal medicine specialists and were established in accordance with current international guidelines. The list of diagnoses for each patient, including comorbidities, was recorded in an electronic database.
The diseases were counted and grouped into typical comorbidity groups as proposed by Charlson (21) and other authors investigating comorbidities in respiratory medicine (22-24).

The laboratory parameters analyzed in this study include serum creatinine, cystatin- $\mathrm{C}$, albumin in urine and other typical biochemistry parameters used for routine comorbidity diagnostics. The eGFR was calculated with CKD-EPI equations (8) using creatinine and cystatin-C. For comorbidity assessment, all physicians were free to review patients' medical files and to order any additional tests including radiology. Values of eGFR $<60 \mathrm{~mL} / \mathrm{min} /$ $1.73 \mathrm{~m}^{2}$ were used as a threshold to separate individuals with altered eGFR (stage 3 a or worse) from individuals with normal or mildly altered kidney function, following KDIGO guidelines (8).

The AASM scoring rules were applied to the polysomnography variables, which included the apneahypopnea index (AHI), the oxygen desaturation index (ODI), mean oxyhemoglobin saturation (mean $\mathrm{SpO}_{2}$ ) and the lowest oxyhemoglobin saturation (lowest $\mathrm{SpO}_{2}$ ) (25). Sleepiness was assessed using a patient-completed questionnaire— the Epworth Sleepiness Scale (ESS).

\section{Statistical analysis}

Variables are expressed as mean (SD) in the case of quantitative variables and as percentages and absolute numbers in the case of qualitative variables. A comparison of dichotomous variables was made using the $\chi^{2}$ test. The Kolmogorov-Smirnov test was used to check the normality of variable distribution. Student's $t$-test was used to examine the difference between the means with normal distribution variables and the Mann-Whitney $U$ test was used when the test of normality failed.

The Pearson or Spearman correlation coefficient was determined for selected laboratory and clinical findings and eGFR $_{\text {cystat }}$.

All results were considered to be statistically significant at $\mathrm{P}<0.05$.

Logistic regression analyses, with eGFR $<60 \mathrm{~mL} / \mathrm{min}$ / $1.73 \mathrm{~m}^{2}$ as the dependent variable were calculated. A backward method was chosen in logistic regression models. Suspected predictors of CKD—age, gender, descriptive data, blood laboratory results, comorbidities and polysomnography variables (i.e., $\mathrm{AHI}, \mathrm{ODI}$, mean $\mathrm{SpO}_{2}$ and lowest $\mathrm{SpO}_{2}$ )—were added to the model.

Statistics analyses were performed using MedCalc 


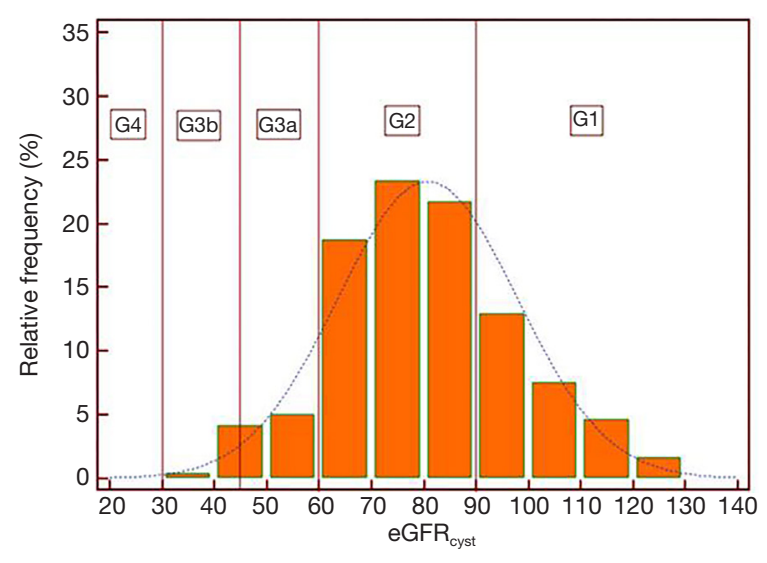

Figure 1 The relative frequency of estimated GFR measurement results when cystatin-C was used as a marker of the GFR $\left(\mathrm{eGFR}_{\text {cystat }}\right.$ ). Plot against normal distribution curve. Chronic kidney disease-epidemiology collaboration group (CKD-EPI) categories added. eGFR $\left(\mathrm{mL} / \mathrm{min} / 1.73 \mathrm{~m}^{2}\right)$. eGFR, estimated glomerular filtration rate.

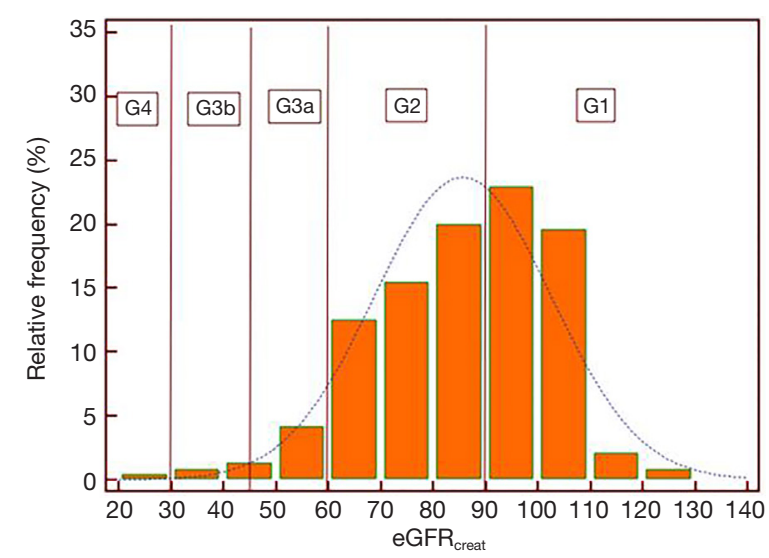

Figure 2 The relative frequency of estimated GFR measurement results when creatinine was used as a marker of GFR (eGFR $\left.\mathrm{creat}_{\text {c }}\right)$. Plot against normal distribution curve. Chronic kidney diseaseepidemiology collaboration group (CKD-EPI) categories added. eGFR $\left(\mathrm{mL} / \mathrm{min} / 1.73 \mathrm{~m}^{2}\right)$. eGFR, estimated glomerular filtration rate.

software 16.2.1 (MedCalc Software, Acacialaan 22, B-8400 Ostend, Belgium).

\section{Results}

Two hundred and forty unselected consecutive patients with confirmed OSA were enrolled in the study: 185 men (77\%) and 55 women $(23 \%)$, with a mean age of 56.8 (SD, 9.9) years and a mean AHI of 38.7 (21.7) events/hour. The mean ESS score was 11.2 (5.7).

\section{How prevalent was reduced eGFR in patients with OSA?}

The total number of patients with reduced eGFR in the OSA group (defined as eGFR $<60 \mathrm{~mL} / \mathrm{min} / 1.73 \mathrm{~m}^{2}$ ) was $23 / 240(9.6 \%)$ when the cystatin-C-based calculation $\left(\mathrm{eGFR}_{\text {cystat }}\right)$ was used and $16 / 240(6.7 \%)$ when the creatinine-based calculation (eGFR $\mathrm{ereat}_{\text {cat }}$ ) was used. The difference was not statistically significant [odds ratio (OR), 1.48; 95\% CI, 0.76-2.88, $\mathrm{P}=0.24]$.

All patients were grouped into categories of eGFR according to the CKD-EPI recommendations. The relative frequency/prevalence of CKD-EPI categories are shown for

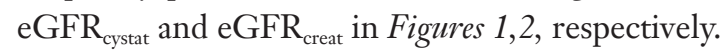

Anthropometrics, sleep and breathing characteristics and main blood biochemistry results in patients with OSA grouped according to eGFR $\mathrm{cystat}_{\text {and }}$ eGFR $\mathrm{R}_{\text {creat }}$ are shown in Tables 1,2, respectively.

\section{What influences eGFR in patients with OSA?}

We observed a clear correlation between $\mathrm{eGFR}_{\text {cystat }}$ and $\mathrm{eGFR}_{\text {creat }}(\mathrm{R}=0.521 ; 95 \% \mathrm{CI}, 0.421-0.6068 ; \mathrm{P}<0.0001)$.

A similar good correlation was observed in patients with eGFR $<60 \mathrm{~mL} / \mathrm{min} / 1.73 \mathrm{~m}^{2}$ ( $\mathrm{R}=0.669 ; 95 \%$ CI, 0.3548 0.8476; $\mathrm{P}=0.0005)$. We tried to assess a link between sleep parameters, such as AHI, ODI, mean $\mathrm{SpO}_{2}$ and lowest $\mathrm{SpO}_{2}$, and eGFR but we did not find any correlations. Significant correlations were found between the $\mathrm{GFR}_{\text {cyst }}$ and pro-brain natriuretic peptide (proBNP) and the total cholesterol (Table 3).

In addition, a weak but statistically significant correlation

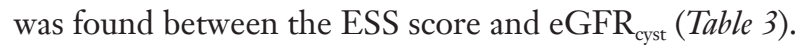

\section{Cross-sectional analysis}

We didn't find any significant differences between eGFR values among patients with OSA grouped by $\mathrm{AHI}$ according to AASM (Table 4).

To assess the influence of BMI on eGFR, we stratified the whole study population by World Health Organization (WHO) BMI categories as underweight, normal, overweight or obese, and compared $\mathrm{eGFR}_{\text {creat }}$ with $\mathrm{eGFR}_{\text {cystat }}$ in those subgroups.

There were no differences in eGFR in non-obese 
Table 1 Patient characteristics in subgroups with a normal and a decreased eGFR based on serum creatinine

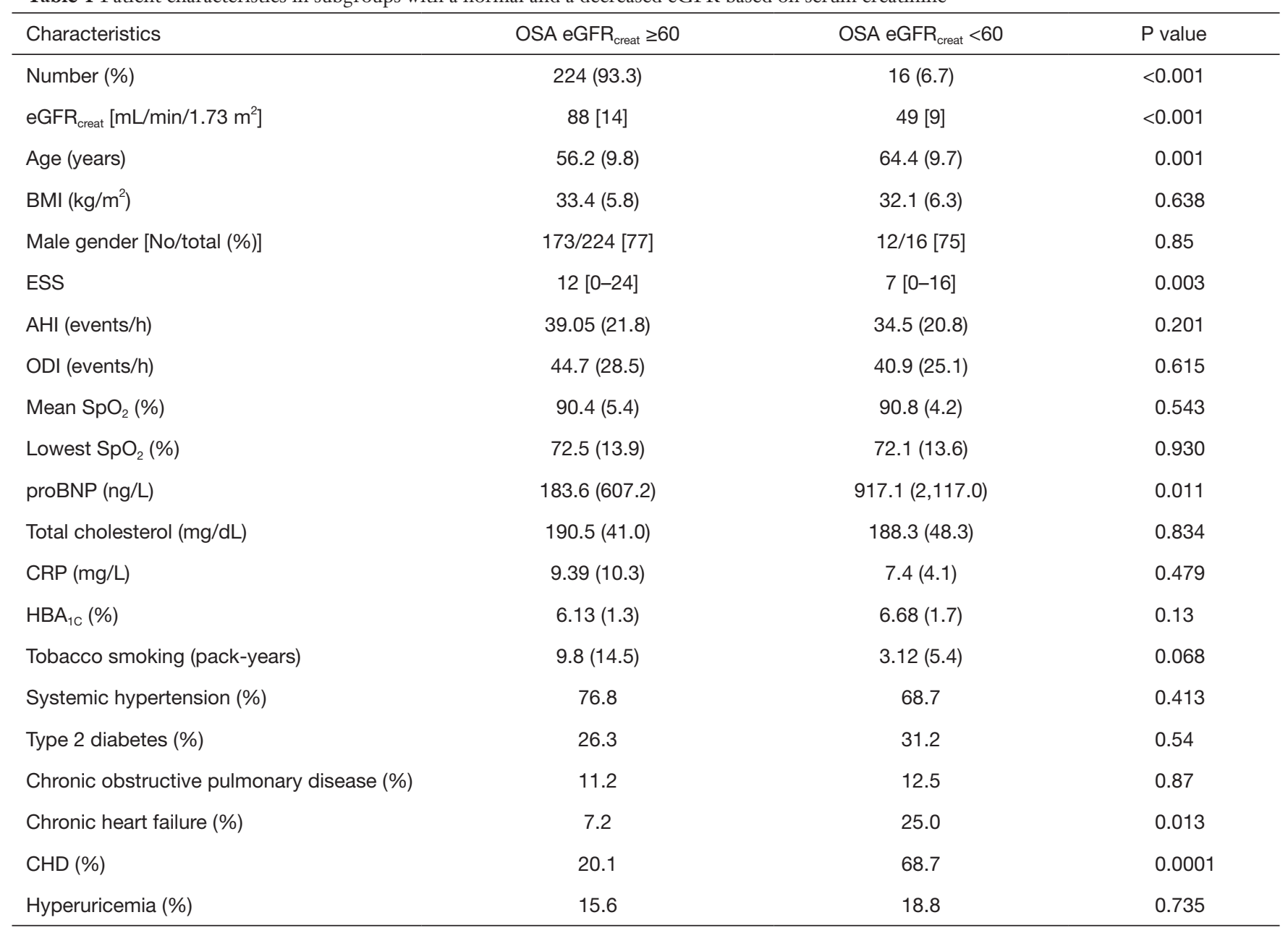

Data expressed as mean (SD), median (min-max) or frequency depending on data type. eGFR, estimated glomerular filtration rate; OSA,

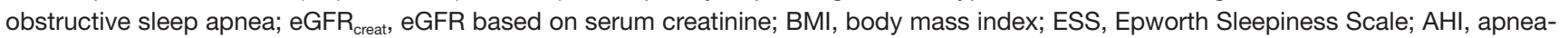
hypopnea index; ODI, oxygen desaturation index; $\mathrm{SpO}_{2}$, oxyhemoglobin saturation; BNP, brain natriuretic peptide; CRP, C-reactive protein; $\mathrm{CHD}$, coronary heart disease.

patients, irrespective of the type of measurement (eGFR $\mathrm{Great}_{\text {t }}$ or eGFR $\left.\mathrm{c}_{\text {cystat }}\right)$. But in obese and extremely obese patients significant differences between $\mathrm{GGFR}_{\text {creat }}$ and eGFR $\mathrm{R}_{\text {cystat }}$ were found. In obese patients with OSA the muscle-dependent eGFR $_{\text {creat }}$ was significantly higher than the muscleindependent $\mathrm{eGFR}_{\text {cystat }}$ (Table 5, Figure 3).

\section{Logistic regression analysis}

In logistic regression analyses, with eGFR $\mathrm{Cystat}<60 \mathrm{~mL} / \mathrm{min} /$ $1.73 \mathrm{~m}^{2}$ as the dependent variable, we found that only BMI, proBNP and coronary heart disease (CHD) were significant (BMI: OR, 1.1; 95\% CI, 1.011-1.196; $\mathrm{P}=0.02$; BNP: 1.06;
95\% CI, 1.001-012; $\mathrm{P}=0.028 ; \mathrm{CHD:}$ OR, 2.7; 95\% CI, $0.96-8.04 ; \mathrm{P}=0.05)$.

\section{Discussion}

The main finding of this study is that there is a significant difference between cystatin-C- and creatinine-based GFR estimations in obese patients with OSA. In our opinion, this observation may influence clinical practice since an increasing number of studies have confirmed CKD as a comorbidity of OSA. There is therefore likely sufficient scientific data to recommend kidney disease screening in patients with newly diagnosed OSA. The following question 
Table 2 Patient characteristics in subgroups with a normal and a decreased eGFR based on serum cystatin-C

\begin{tabular}{|c|c|c|c|}
\hline Characteristics & OSA eGFR cystat $\geq 60$ & OSA eGFR cystat $_{1}<60$ & $P$ value \\
\hline Age (years) & $55.8(9.6)$ & $65.4(8.2)$ & $<0.001$ \\
\hline eGFR $\operatorname{cystat}\left(\mathrm{mL} / \mathrm{min} / 1.73 \mathrm{~m}^{2}\right)$ & 84 [15] & $51[7]$ & $<0.001$ \\
\hline $\mathrm{BMI}\left(\mathrm{kg} / \mathrm{m}^{2}\right)$ & $33.2(5.8)$ & $34.4(6.2)$ & 0.359 \\
\hline ESS & $11[0-24]$ & $8[0-17]$ & 0.013 \\
\hline AHI (events/h) & $38.4(21.4)$ & $41.5(24.5)$ & 0.521 \\
\hline ODI (events/h) & $43.9(27.5)$ & $49.4(34.2)$ & 0.372 \\
\hline Mean $\mathrm{SpO}_{2}(\%)$ & $90.4(5.5)$ & $91.0(3.6)$ & 0.271 \\
\hline Total cholesterol (mg/dL) & $192.2(41.7)$ & $173.6(35.4)$ & 0.04 \\
\hline $\mathrm{CRP}(\mathrm{mg} / \mathrm{L})$ & $9.2(10.9)$ & $8.6(5.2)$ & 0.813 \\
\hline $\mathrm{HBA}_{1 \mathrm{C}}(\%)$ & $6.14(1.3)$ & $6.4(1.4)$ & 0.242 \\
\hline Tobacco smoking (pack-years) & $9.73(14.4)$ & $6.1(11.9)$ & 0.251 \\
\hline Systemic hypertension (\%) & 75.1 & 87.0 & 0.2799 \\
\hline Type 2 diabetes (\%) & 24.4 & 47.5 & 0.0339 \\
\hline Chronic obstructive pulmonary disease (\%) & 11.1 & 13.0 & 0.816 \\
\hline Chronic heart failure (\%) & 7.2 & 21.0 & 0.0514 \\
\hline
\end{tabular}

Data expressed as mean (SD), median (min-max) or frequency depending on data type. eGFR, estimated glomerular filtration rate; OSA,

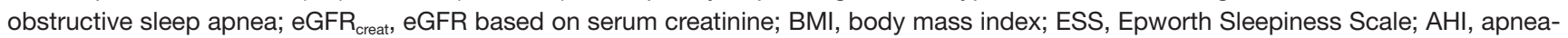
hypopnea index; ODI, oxygen desaturation index; $\mathrm{SpO}_{2}$, oxyhemoglobin saturation; BNP, brain natriuretic peptide; CRP, C-reactive protein; $\mathrm{CHD}$, coronary heart disease.

is important to discuss: is creatinine-based eGFR assessment sufficient for such screening? From a clinician's perspective, creatinine-based estimations have several advantages. Serum creatinine, which is traditionally used as a biomarker for renal function, is inexpensive and broadly used. However, it can be hugely affected by extra-renal factors, especially abnormally high body muscle mass or body size. According to KDIGO, in specific groups of patients, such as those with OSA, serum creatinine is occasionally underestimated or overestimated. Cystatin-C is a biomarker potentially less affected by muscle and that likely reflects renal function more sensitively than serum creatinine in very obese patients. As patients with OSA are characterized by a high BMI, patient mass depends mainly on fat mass. In such cases potential error linked to non-linearity or non-reliability of creatinine-based eGFR equations in individuals with extremely high BMI needs to be considered. In our study we have shown that the number of patients with OSA with suspected CKD was higher when the cystatin-C measurement was added (6.7\% vs. 9.6\%). In addition, we have shown that patients with OSA with a BMI over $30 \mathrm{~kg} / \mathrm{m}^{2}$ need to be assessed with the use of cystatin-C measurements, due to the possible underestimation of CKD with classical creatinine testing. In our group very obese patients showed lower mean $\mathrm{GFR}_{\text {cystat }}$ than $\mathrm{eGFR}$ creat, confirming the non-reliability of CKD-EPI estimations in patients with extreme body mass, as suggested by CKD- 
Table 3 Correlation between selected laboratory and clinical findings and eGFR based on cystatin-C

\begin{tabular}{lcc}
\hline Parameters & $P$ value & Correlation coefficient \\
\hline proBNP & 0.0053 & -0.247 \\
Total cholesterol & 0.0438 & 0.13 \\
Age & $<0.0001$ & -0.526 \\
Creatinine & $<0.0001$ & -0.277 \\
ESS & 0.0121 & 0.162 \\
Minimum $\mathrm{SpO}_{2}$ & 0.833 & 0.014 \\
ODI & 0.9868 & 0.001 \\
Mean SpO & \\
AHI & 0.9915 & 0.001 \\
BMI & 0.2004 & 0.083 \\
Triglyceride & 0.352 & 0.06 \\
CRP & 0.0516 & 0.126 \\
$\mathrm{HbA}$ & 0.5249 & -0.047 \\
Smoking (pack-years) & 0.129 & -0.064 \\
\hline
\end{tabular}

eGFR, estimated glomerular filtration rate; $\mathrm{BNP}$, brain natriuretic peptide; ESS, Epworth Sleepiness Scale; $\mathrm{SpO}_{2}$, oxyhemoglobin saturation; ODI, oxygen desaturation index; AHI, apneahypopnea index; BMI, body mass index; CRP, C-reactive protein.
EPI group experts. We have also tried to look at what type of comorbidities are important and could be used to guide clinicians thinking of adding cystatin-C measurements to the test panel for patients with OSA. In both correlation and regression tests we have found that patients with CHD are better suited to cystatin-C testing. In our study, we also found an increase in proBNP in patients with diminished eGFR and a correlation between eGFR $\mathrm{R}_{\text {cystat }}$ and proBNP. This finding may confirm recent data suggesting the role of cystatin- $\mathrm{C}$ as an emerging biomarker in cardiovascular diseases (26) or confirm the well-known data showing the role of kidney diseases in atherogenesis.

The discussion about screening for reduced eGFR in patients with OSA needs to also focus on the next question: how prevalent is reduced eGFR or CKD in patients with OSA, and is such coexistence of clinical importance?

OSA is frequently associated with other diseases. Hypertension, diabetes and obesity are typical examples of OSA comorbidities. CKD was suspected to be a comorbidity of OSA, but the pathophysiological and epidemiological interrelation between CKD and OSA is still unclear (1). Hanly et al. proposed that nocturnal hypoxemia may contribute to the pathogenesis of CKD and its progression to kidney failure. In addition, chronic intrarenal hypoxia may result in tubulointerstitial injury (1). Moreover, there

Table 4 eGFRs based on creatinine and cystatin-C in subgroups of patients with obstructive sleep apnea divided by AHI severity

\begin{tabular}{lcccc}
\hline Parameters & $\mathrm{AHI}<15$ events/hour & AHI 15-30 events/hour & AHI $>30$ events/hour & P value for trend \\
\hline eGFR $_{\text {creat }}\left(\mathrm{mL} / \mathrm{min} / 1.73 \mathrm{~m}^{2}\right)$ & $83[15]$ & $84[18]$ & $86[16]$ & $\mathrm{NS}$ \\
eGFR $_{\text {cystat }}\left(\mathrm{mL} / \mathrm{min} / 1.73 \mathrm{~m}^{2}\right)$ & $80[15]$ & $79[17]$ & $81[17]$ & $\mathrm{NS}$ \\
\hline
\end{tabular}

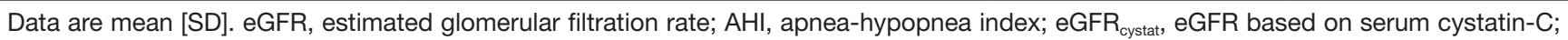
eGFR $_{\text {creat }}$, eGFR based on serum creatinine.

Table 5 The mean results of GFR estimations using creatinine and cystatin-C in the whole study group stratified according the WHO BMI categories

\begin{tabular}{|c|c|c|c|c|c|}
\hline Parameters & $\mathrm{BMI}<18.5 \mathrm{~kg} / \mathrm{m}^{2}$ & BMI $18.5-25 \mathrm{~kg} / \mathrm{m}^{2}$ & BMI $25-30 \mathrm{~kg} / \mathrm{m}^{2}$ & BMI $30-40$ kg/m² & $\mathrm{BMI}>40 \mathrm{~kg} / \mathrm{m}^{2}$ \\
\hline $\mathrm{eGFR}_{\text {cystat }}\left(\mathrm{mL} / \mathrm{min} / 1.73 \mathrm{~m}^{2}\right)$ & NA & 80 [16] & $83[18]$ & $80[17]$ & 78 [17] \\
\hline$P$ value & NA & 0.766 & 1.0 & 0.0195 & 0.0164 \\
\hline
\end{tabular}

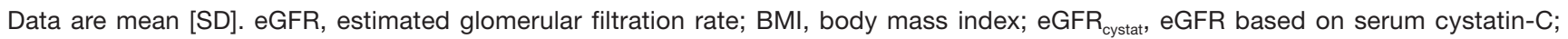
eGFR $_{\text {creat }}$, eGFR based on serum creatinine. 


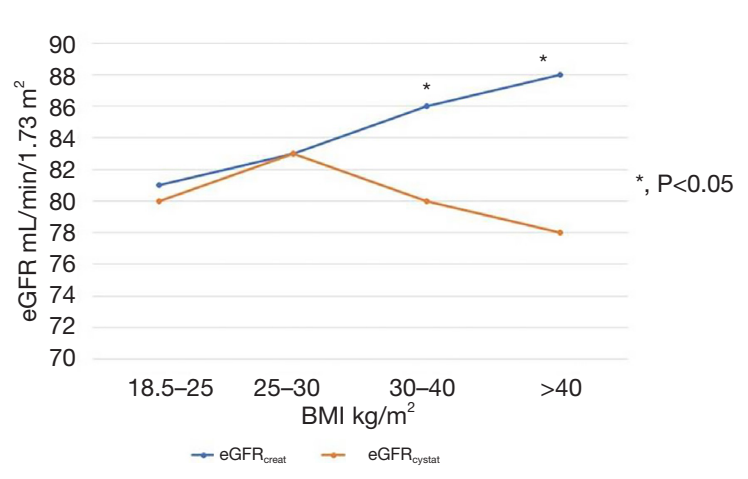

Figure 3 The mean results of GFR estimations using creatinine $\left(\mathrm{eGFR}_{\text {creat }}\right)$ and cystatin-C $\left(\mathrm{eGFR}_{\text {cystat }}\right)$ in the whole study group stratified according to the WHO BMI categories. GFR, glomerular filtration rate. BMI, body mass index; eGFR, estimated glomerular filtration rate.

are also data suggesting that chronic kidney hypoxia is a common final pathway in end-stage renal disease (2).

From a clinical point of view the relationships between CKD and OSA are of great interest due to potential epidemiological and clinical problems. The epidemiological relationships between OSA and CKD may be studied in different ways.

In our study, the prevalence of reduced GFR, defined as eGFR $<60 \mathrm{~mL} / \mathrm{min} / 1.73 \mathrm{~m}^{2}$, varied from $6.7 \%$ to $9.6 \%$ depending on the type of biomarker used. When traditional creatinine measurements were used for eGFR estimation, the prevalence of CKD was lower (6.7\%), and when the newer cystatin- $\mathrm{C}$ measurement was used, it was higher (9.6\%). Such a difference suggests that the use cystatin-C instead of creatinine may considerately influence the assessment of disease prevalence. Most other cohort OSA studies have used only one type of biomarker, most often creatinine, for CKD assessments. Marrone and collaborators gathered patients with OSA from a few European countries from the European Sleep Apnea Database (ESADA) group to study the prevalence of CKD. Similarly to our study, Marrone et al. used the same contemporary CKD-EPI equations. When creatinine was used as a biomarker the prevalence of CKD was found to be $6.1 \%$ (5). Across other cohort studies in patients with OSA a great variability in CKD prevalence can be seen. Reported numbers are hugely influenced by the methodology used. Kanbay et al. used the Cockcroft-Gault formula, and detected a significant GFR decrease when the severity of OSA increased (27). Chou et al. studied 40 patients referred for polysomnography to rule out sleep apnea and used a Modification of Diet in Renal Disease (MDRD) formula for GFR estimation. The prevalence of CKD in patients with OSA was 14\% (12). Iseki et al. evaluated patients in a OSA syndrome registry of CKD, defined as an eGFR $<60 \mathrm{~mL} / \mathrm{min} / 1.73 \mathrm{~m}^{2}$, using MDRD and creatinine, and found a $30.5 \%$ CKD prevalence in patients with sleep apnea (14). Fleischmann et al. used the same MDRD equation and creatinine measurement for determination of eGFR in 158 consecutive patients with OSA. They found that $11.4 \%$ of patients with OSA had eGFR $<60 \mathrm{~mL} / \mathrm{min} / 1.73 \mathrm{~m}^{2}$ (13).

Very few OSA studies have used cystatin-C to determine CKD prevalence. Zhang et al. studied cystatin-C levels in patients with OSA, concluding that serum cystatin-C was associated with the severity of OSA in younger men (17). In a later study after introduction of continuous positive airway pressure (CPAP) treatment, the same authors concluded that CPAP can decrease cystatin-C levels among patients with severe OSA and may prevent latent renal impairment (28). Kato et al. studied 267 consecutive patients with OSA referred for polysomnography (29). The authors concluded that severe OSA independently increases serum cystatin-C levels in patients without CKD and that cystatin-C needs to be considered as a biomarker that reflects both clinically latent renal dysfunction and cardiovascular risk, which are influenced by OSA.

Our study has some limitations. First, our study is a single-center cross-sectional analysis. Second, the population we studied was not entirely comparable to the other populations. However, we studied consecutive and unselected patients, and therefore think that we studied a typical Polish population affected by OSA.

What's new?

In our study we discussed the role of creatinine- and cystatin-C-based estimations of eGFR in the assessment of eGFR in patients with OSA.

\section{Conclusions}

GFR in OSA can be routinely screened by using creatininebased estimations (eGFR $\mathrm{ereat}_{\text {cat }}$ ). In a selected group of patients with OSA with BMI over $30 \mathrm{~kg} / \mathrm{m}^{2}$ the addition of cystatin-C for assessment of eGFR is suggested.

\section{Acknowledgments}

Part of this article has been presented as an abstract at the European Respiratory Society Annual Congress (ERS). 
Funding: The study was fully funded by National Institute of Tuberculosis and Lung Diseases, Warsaw, Poland. The funders had no role in study design, data collection and analysis, decision to publish, or preparation of the manuscript. No additional funding was received for this research.

\section{Footnote}

Conflicts of Interest: The authors have no conflicts of interest to declare.

Ethical Statement: The authors are accountable for all aspects of the work in ensuring that questions related to the accuracy or integrity of any part of the work are appropriately investigated and resolved. All procedures performed in studies involving human participants and written participant consents were in accordance with the ethical standards of the institutional and/or national research committee; Committee of National Institute of Tuberculosis and Lung Diseases in Warsaw and with the 1964 Helsinki declaration and its later amendments or comparable ethical standards. The study was approved by the local Ethics Committee of the Institute of Tuberculosis and Lung Diseases (No. KB-57).

Open Access Statement: This is an Open Access article distributed in accordance with the Creative Commons Attribution-NonCommercial-NoDerivs 4.0 International License (CC BY-NC-ND 4.0), which permits the noncommercial replication and distribution of the article with the strict proviso that no changes or edits are made and the original work is properly cited (including links to both the formal publication through the relevant DOI and the license). See: https://creativecommons.org/licenses/by-nc-nd/4.0/.

\section{References}

1. Hanly PJ, Ahmed SB. Sleep apnea and the kidney: is sleep apnea a risk factor for chronic kidney disease? Chest 2014;146:1114-22.

2. Fine LG, Norman JT. Chronic hypoxia as a mechanism of progression of chronic kidney diseases: from hypothesis to novel therapeutics. Kidney Int 2008;74:867-72.

3. Torres G, Sánchez-de-la-Torre M, Barbé F. Relationship between OSA and hypertension. Chest 2015;148:824-32.

4. Reutrakul S, Mokhlesi B. Obstructive sleep apnea and diabetes: a state of the art review. Chest 2017;152:1070-86.
5. Marrone O, Battaglia S, Steiropoulos P, et al. Chronic kidney disease in European patients with obstructive sleep apnea: the ESADA cohort study. J Sleep Res 2016;25:739-45.

6. Shlipak MG, Matsushita K, Ärnlöv J, et al. Cystatin C versus creatinine in determining risk based on kidney function. N Engl J Med 2013;369:932-43.

7. Levin A, Stevens PE. Summary of KDIGO 2012 CKD Guideline: behind the scenes, need for guidance, and a framework for moving forward. Kidney Int 2014;85:49-61.

8. Summary of Recommendation Statements. Kidney Int Suppl (2011) 2013;3:263-5.

9. Mathew R, Castriotta RJ. High hypopnea/apnea ratio (HAR) in extreme obesity. J Clin Sleep Med 2014;10:391-6.

10. Kuźnar-Kamińska B, Grabicki M, Trafas T, et al. Body composition, anthropometric indices and hydration status of obstructive sleep apnea patients: can cachexia coexist with obesity? In: Pokorski M. Clinical research and practice. Cham: Springer, 2017:43-51.

11. Ahmed SB, Ronksley PE, Hemmelgarn BR, et al. Nocturnal hypoxia and loss of kidney function. PLoS One 2011;6:e19029.

12. Chou YT, Lee PH, Yang CT, et al. Obstructive sleep apnea: a stand-alone risk factor for chronic kidney disease. Nephrol Dial Transplant 2011;26:2244-50.

13. Fleischmann G, Fillafer G, Matterer H, et al. Prevalence of chronic kidney disease in patients with suspected sleep apnoea. Nephrol Dial Transplant 2010;25:181-6.

14. Iseki K, Tohyama K, Matsumoto T, et al. High Prevalence of chronic kidney disease among patients with sleep related breathing disorder (SRBD). Hypertens Res 2008;31:249-55.

15. Uyar M, Davutoğlu V, Gündoğdu N, et al. Renal functions in obstructive sleep apnea patients. Sleep Breath 2016;20:191-5.

16. Petrov ME, Kim Y, Lauderdale DS, et al. Objective sleep, a novel risk factor for alterations in kidney function: the CARDIA study. Sleep Med 2014;15:1140-6.

17. Zhang XB, Lin QC, Deng CS, et al. Elevated serum cystatin $\mathrm{C}$ in severe OSA younger men without complications. Sleep Breath 2013;17:235-41.

18. Canales MT, Taylor BC, Ishani A, et al. Reduced renal function and sleep-disordered breathing in communitydwelling elderly men. Sleep Med 2008;9:637-45.

19. Pływaczewski R, Brzecka A, Bielicki P, et al. Sleep related breathing disorders in adults - recommendations of Polish Society of Lung Diseases. Pneumonol Alergol Pol 
2013;81:221-58.

20. Sin DD, Anthonisen NR, Soriano JB, et al. Mortality in COPD: Role of comorbidities. Eur Respir J 2006;28:1245-57.

21. Charlson ME, Pompei P, Ales KL, et al. A new method of classifying prognostic comorbidity in longitudinal studies: development and validation. J Chronic Dis 1987;40:373-83.

22. Barnes PJ, Celli BR. Systemic manifestations and comorbidities of COPD. Eur Respir J 2009;33:1165-85.

23. Soriano JB, Visick GT, Muellerova H, et al. Patterns of comorbidities in newly diagnosed COPD and asthma in primary care. Chest 2005;128:2099-107.

24. Antonelli Incalzi R, Fuso L, De Rosa M, et al. Comorbidity contributes to predict mortality of patients with chronic obstructive pulmonary disease. Eur Respir J 1997;10:2794-800.

25. Kapur VK, Auckley DH, Chowdhuri S, et al. Clinical

Cite this article as: Nowiński A, Czyżak-Gradkowska A, Jonczak L, Korzybski D, Peradzyńska J, Pływaczewski R, Śliwiński P. Glomerular filtration rate in patients with obstructive sleep apnea: the influence of cystatin-C-based estimations and comorbidity. J Thorac Dis 2020;12(3):175-183. doi: $10.21037 /$ jtd.2020.02.11 practice guideline for diagnostic testing for adult obstructive sleep apnea: an American Academy of Sleep Medicine Clinical Practice Guideline. J Clin Sleep Med 2017;13:479-504.

26. Angelidis C, Deftereos S, Giannopoulos G, et al. Cystatin $\mathrm{C}$ : an emerging biomarker in cardiovascular disease. Curr Top Med Chem 2013;13:164-79.

27. Kanbay A, Buyukoglan H, Ozdogan N, et al. Obstructive sleep apnea syndrome is related to the progression of chronic kidney disease. Int Urol Nephrol 2012;44:535-9.

28. Zhang XB, Jiang XT, Lin QC, et al. Effect of continuous positive airway pressure on serum cystatin $\mathrm{C}$ among obstructive sleep apnea syndrome patients. Int Urol Nephrol 2014;46:1997-2002.

29. Kato K, Takata Y, Usui Y, et al. Severe obstructive sleep apnea increases cystatin $\mathrm{C}$ in clinically latent renal dysfunction. Respir Med 2011;105:643-9. 